\title{
The Investigation of Cell Culture Conditions to Maintain Chicken Embryonic Stem Cells as Totipotent Cells
}

\author{
Du Lixin* and An Jing \\ Lab of Animal Biotech, College of Animal Science Technolojgy, Shandong Agriculture University, Taian, 271018, China
}

\begin{abstract}
The ES cell can provide a useful system for studying differentiation and development in vitro and a powerful tool for producing transgenic animalds. To investigate the culture condition of chicken embryonic stem (CES) cells which can retain their multipotentiality or totipotency, three kinds of feeder layer cells, SNL cells, primary mice embryonic fibroblasts (PMEF) cells and primary chicken embryonic fibroblasts (PCEF) cells, were used as the feeder cells in media of DMEM supplemented with leukemia inhibitory factor (LIF), basic fibroblast growth factor (bFGF) and stem cell factor (SCF) for co-culture with blastoderm cells from stage $\mathrm{X}$ embryos of chicken. The alkaline phosphatase (AKP) test, differentiation experiment in vitro and chimeric chicken production were carried out. The results showed that culture on feeder layer of PMEF yielded high quality CES cell colonies. The typical CES cells clone shape revealed as follows: nested aggregation (clone) with clear edge and round surface as well as close arrangement within the clone. Strong alkaline phosphatase (AKP) reactive cells were observed in the fourth passage cells. On the other hand, the fourth passage CES cells could differentiate into various cells in the absence of feeder layer cells and LIF in vitro. The third and fourth passage cells were injected into the subgerminal cavity of recipient embryos at stage X. Of 269 Hailan embryos injected with CES cells of Shouguang Chickens, $8.2 \%$ (22/269) survived to hatching, 5 feather chimeras had been produced. This suggests that an effective culture system established in this study can promote the growth of CES cells and maintain them in the state of undifferentiated and development, which lays a solid foundation for the application of CES cells and may provide an alternative tool for genetic modification of chickens. (AsianAust. J. Anim. Sci. 2003. Vol 16, No. 8 : 1102-1107)
\end{abstract}

Key Words : Embryonic Stem Cells, Feeder Layer Cells, Chicken ES Cells, Chimeras

\section{INTRODUCTION}

Embryonic stem (ES) cells which are derived from the blastocyst stage of the embryo have the same characteristics as embryo cells They are capable of differentiating into all types of cells and can be isolated and cultured for long-term in vitro. These cultured cells can participate in the development of all cell lineages including the germ line when they are implanted into host blastocysts. ES cells were first isolated by culturing of inner cell mass of preimplantation embryo in mouse (Evans and Kaufman, 1981; Nichols et al., 1990). To date cultures of ES cells or ES-like cells have been developed from pig (Wheeler et al., 1994), bovine (First et al., 1994), sheep (Piedrahita et al., 1999), rabbit (Giles et al., 1993), zebra fish (Sun et al., 1995) and monkey (Thomson et al., 1995), human (Thomson et al., 1998; Shamblott et al., 1998) etc.

In avian species, Petittes et al. (1990); Natio et al. (1991); Thoraval et al. (1994); One et al. (1994); Kino et al. (1997) have demonstrated that cells derived from the early chicken blastoderm will contribute to the somatic and the germ line when injected into recipient embryos to form chimeras. Etches et al. (1997); Du Lixin (2002) had maintained these blastodermal cells in vitro for $48 \mathrm{~h}$ and

\footnotetext{
* Corresponding Author: Du Lixin, Institute of Animal Science, Chinese Academy of Agricultural Sciences Beijing 100094, China, Tel: +86-10-62819997, E-mail: 1xdu@263.net

Received November 14, 2002; Accepted April 4, 2003
}

proven that genetically modified avian ES cells continued to have the ability to join in the somatic and the germ line. Pain et al. (1996) first reported that avian stem cells with multiple morphogenetic potentialities were derived and maintained by long-term culture $(160 \mathrm{~d})$ of blastodermal cells in vitro and two chickens that injected 7 day cultured chicken embryonic cells had been proven as germ-line chimeras. However, there are a lot of uncertain factors on the culture of CES cells, such as feeder layer and culture condition. The aim of the present study was to compare the effect of three feeder layer cells, three cytokines and other factors on the growth of chicken ES cells in order to set up the optimum culture condition allowing the growth and characterization of chicken ES cells.

\section{MATERIALS AND METHODS}

\section{Experimental animal}

KM mice were got from the experimental animal center of the Biological Production Institution in Shandong Taian; Shouguang Chickens (Pigment genotype: CCOOEE) were provided by Liaocheng Jiaming Breeding Chicken Farm; Hailan eggs (Pigment genotype: IICCOO) were got from Shandong Taian Breeding Chicken Farm.

\section{Medium}

ES Cells Medium was composed of DMEM (High glucose, Gibco) containing 10\% foetal bovine serum 
(Gibco), 2\% chicken serum (Gibco), $0.1 \mathrm{mM} \quad \beta$ mercaptoethanol (Gibco), $100 \mathrm{U} / \mathrm{ml}$ penicillin, $100 \mu \mathrm{g} / \mathrm{ml}$ streptomycin, $10 \mathrm{ng} / \mathrm{ml} \mathrm{bFGF} \mathrm{(Gibco),} 5 \mathrm{ng} / \mathrm{ml} \mathrm{SCF}$ (LTD), 1000 IU/ml LIF (Sigma).

Somatic Cells Medium was composed of DMEM (high glucose, Gibco) containing 10\% FCS (Baian).

\section{Preparation of embryonic fibroblast cells}

Primary chicken embryonic fibroblast (PCEF) cells and primary mice embryonic fibroblast (PMEF) cells were made according to the method of Gao Shuping et al. (2000) in our lab. SNL cells were kindly provided by Dr Zhengruizhen.

\section{Preparation of feeder layer cells}

The 3rd-6th passage PCEF cells and PMEF cells were selected to make feeder layer cells. These cells were treated with DMEM containing $10 \mu \mathrm{g} / \mathrm{ml}$ Mitomycin -C (Sigma) for $1.5-2 \mathrm{~h}$ at $37^{\circ} \mathrm{C}$, then washed no less than 5 times using PBS. Dispersed single cells $\left(5 \times 10^{5}\right.$ cells $\left./ \mathrm{ml}\right)$ were incubated on dishes (Nunc), which were coated with $0.1 \%$ gelatin (German) solution. SNL feeder layer cells were not confined by their cells passages and the operating procedure is same as above. SNL cells were used at $2 \times 10^{5}$ cells $/ \mathrm{ml}$ as feeder. The feeder layer cells could be used as long as the monolayer cells were formed.

\section{Culture of chicken blastdermal cells at stage $X$}

Freshly laid unincubated eggs of Shouguang chickens were used. Only embryos at stage IX-XI were used to prepare the blastodermal cells. The stages of the chicken embryos were estimated according to Eyal-Giladi and Kochav (1976). The entire blastoderm was removed by gentle aspiration with a Pasteur Pipette in PBS at room temperature then centrifuged at 1,000 rpm for $10 \mathrm{~min}$. The cell pellet was then slowly mechanically dissociated in ES cells medium. Single cells were seeded in ES cells medium on feeder layer cells at a final concentration of one embryo per ml medium.

These cells were maintained at $37^{\circ} \mathrm{C}$ in $5 \% \mathrm{CO}_{2}$ and $90 \%$ humidity. If the cells were scarcity some newly fresh blastodermal cells could be added in the third day. The medium was changed partially $(50 \%)$ every day. When colonies formed, these were removed, incubated in $0.25 \%$ trypsin (Gibco) and dispersed by gentle aspiration. Dissociated single cells or smaller colonies were seeded on new feeder layer cells for subculture.

\section{Alkaline phosphatase reaction}

After washing, cultured chicken blastodermal cells were fixed with cold $1 \%$ paraformaldehyde for $15 \mathrm{~min}$ at $4^{\circ} \mathrm{C}$ Alkaline phosphatase staining solution (BCIP/NBT) (Shanghai Sangon) was added after cells were washed with
PBS. The reaction was incubated for $1.5 \mathrm{~h}$ at $37^{\circ} \mathrm{C}$, stopped by addition of $10 \mathrm{mM}$ EDTA and the cells were washed with PBS. Coloured colonies were scored using an inverted microscope (Leica).

\section{In vitro differentiation}

To examine the differentiation ability of these cultured cells, colonies were gently agitated and washed with PBS. These cells were re-suspended in ES cells medium without mLIF and seeded on feeder layer cells or on gelatin-coated dishes without feeder layer cells. The medium was changed partly $(50 \%)$ every day and morphology was monitored daily.

\section{Production of chimeras}

Stage X White Hailan embryos from newly laid eggs were used as recipient embryos, the third and the fourth passage Shouguang CES cells as donors. After washing with PBS, the colonies were incubated with $0.25 \%$ trypsin then centrifuged at 1,000 rpm for 8-10 min. The cell pellet was collected and slowly mechanically dissociated in PBS. A small window was made on the lateral part of the egg and the shell membrane was removed. Approximated $2 \mu \mathrm{l}$ of cell suspension at 200 cells/ $\mu \mathrm{l}$ was injected into the subgerminal cavity using a new micropipette $(60-80 \mu \mathrm{l})$ for every egg. The egg was transferred into a surrogate shell I derived from an egg $25 \mathrm{~g}$ bigger than the original one. Supplemented with egg white the window was sealed with two layers of shell membrane. During the first $72 \mathrm{~h}$ the eggs were incubated at a temperature of $38.2^{\circ} \mathrm{C}$, rotated through $90^{\circ}$ per hour. At the third day the well developed embryos were transferred into another surrogate shell II, supplemented with egg white. The sealed eggs were incubated until the 21 st day with rotation of $30^{\circ}$ per hour, at a temperature of $38.2^{\circ} \mathrm{C}$.

\section{RESULTS}

\section{Growth of chicken ES cells}

Growth was started from chicken blastoderms collected at stage X (Eyal-Giladi and Kochar, 1976) that were known to contain cells that contribute to the germ line in chimeras (Carsience et al., 1993; Petitte et al., 1990) in an attempt to develop cultures of chicken totipotent embryonic cells (potentially ES cells), After cultured for $24 \mathrm{~h}$ the CB cellcolonies composed of 10-20 cells appeared on the feeder layer cells and these colonies became more obvious after another 24 h. 3-4 days later we could see some nest-like, hill-like, sunflower-like colonies and 5 days later these colonies was bigger and the edge was clearer but there were also some differentiated cells. Thus it was the time to reproduce; dispersed cells seeded on new feeder layer cells could proliferate quickly. 
(\%)

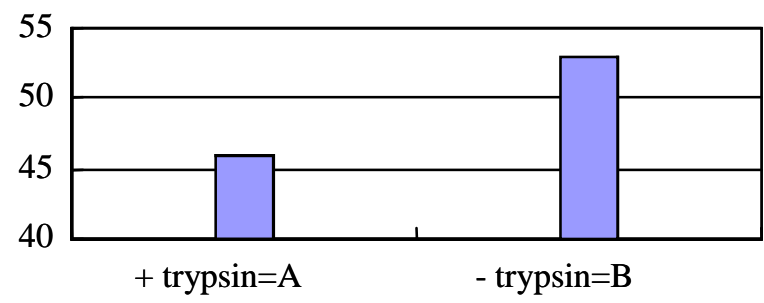

Figure 1. Effect of trypsin on percentage of AKP positive colonies in primary culture.

(\%)

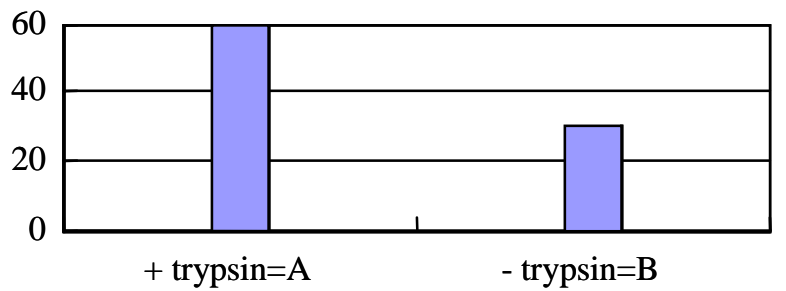

Figure 2. Effect of trypsin on percentage of AKP positive colonies in subculture.

\section{Effect of feeder layer cells on ces growth}

In this study we used PCEF cells, PMEF cells and SNL cells respectively to make feeder layer cells and then observed the growth behavior of the CB cells. The result indicated that the most effective one for the growth of the CB cells was PMEF cells not only in primary culture but also in subculture. The fourth passage CES cells were gained by using PMEF cells as feeder cells. SNL feeder cells also had the ability to sustain CES cells but they shrank and came off easily from the dishes after 4 days. PCEF feeder cells could last a longer time but CES colonies on the PCEF feeder layer cells were more readily differentiated.

\section{Effect of cell-dispersed liquid on the growth of CES cells}

Cell-dispersed liquid such as trypsin could stimulate the dispersion of cells; $0.25 \%$ trypsin as cell-dispersed liquid was used when we treated CB cells in this study. The result showed that percentage of alkaline phosphatase positive of B (no added trypsin) was significantly higher than that of A (added trypsin) in primary culture (Figure 1). While in subculture the result was reversed (Figure 2).

\section{Effect of cytokines on the growth of CES cells}

Chicken blastodermal cells were plated at a final concentration of one embryo/ml in ES cells medium on PMEF feeder layer cells in order to define the growth factor and cytokines requirement for proliferation of alkaline phosphatase positive cells. Different growth factors were added to the medium as indicated in Figure 3. After four days of culture, cells were fixed and stained for alkaline phosphatase activity and the positive colonies with a
A: h-LIF, B: mSCF, C: bFGF,

D: h-LIF+mSCF+bFGF

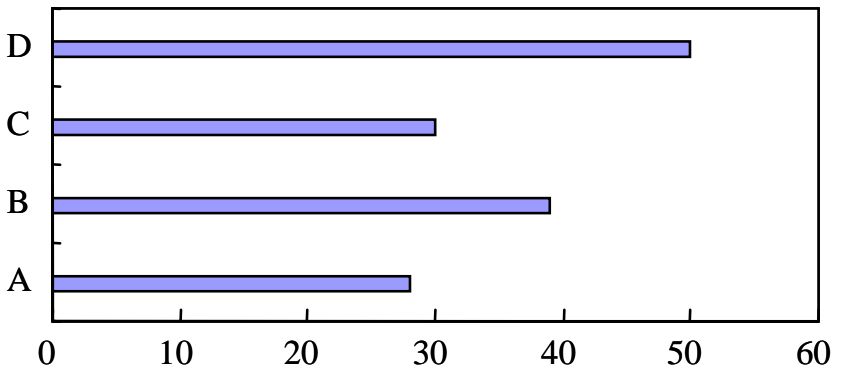

Figure 3. Effect of cytokine on percentage of AKP positive colonies.

A: area pellucida, B: area opaca,

C: area pellucida and opaca

(\%)

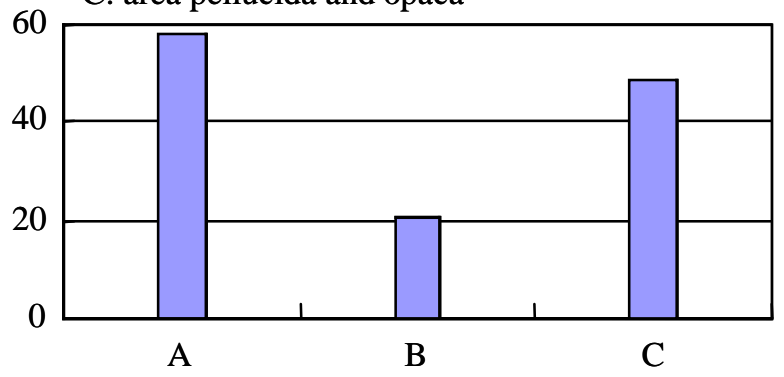

Figure 4. Effect of area pellucida or opaca on percentage of AKP positive colonies.

compact and round morphology were scored. The result showed that mSCF, bFGF could both stimulate the proliferation of CES cells and h-LIF could inhibit the differentiation of cells. None of the factors significantly increased the number of positive colonies when added alone. However the combination of h-LIF, bFGF and mSCF highly significantly enhanced percentage of alkaline phosphatase positive colonies.

In this work only embryos at stage IX-XI could be used to derive CES cells. The chicken embryos were estimated to be at the equivalent stage $\mathrm{X}$ to $\mathrm{XI}$ at laying. At this stage two distinct areas of the blastoderm became evident when viewed dorsally. One was the more transparent central area pellucida that overlies the subgerminal space and the other one was the more dense area opaca that consists of the peripheral blastodermal cells that have remained in contact with the yolk. The region at the border of these two areas was the marginal zone (MZ). The area pellucida and the area opaca were separated and cultured. The result showed that percentage of alkaline phosphatase positive colonies of area pellucida was significantly higher than that of area opaca. (Figure 4). 


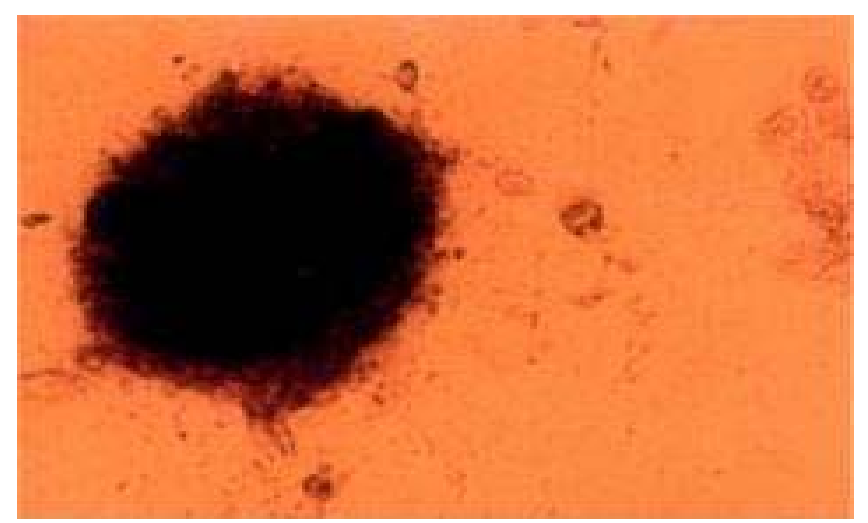

Figure 5. Alkaline phosphatase positive colonies (100×)

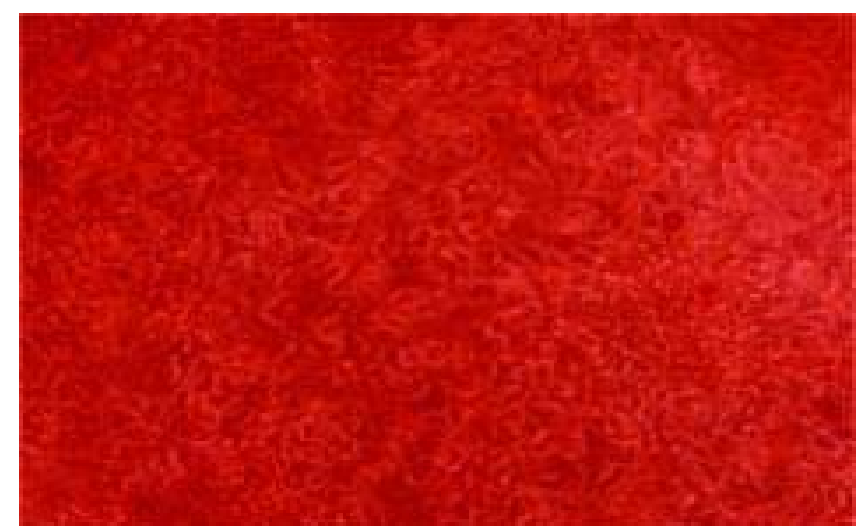

Figure 6. Spontaneously differentiated cells (250×).

\section{Result of AKP staining}

Alkaline phosphatase had been shown previously to be a marker of mouse ES cells and teratocarcinoma cells. CES cells also had the alkaline phosphatase activity. As shown in Figure 5 the fourth passage CES cells stained by BCIP/NBT solution presented dark blue.

The ability to differentiate into simple or complex embryoid bodies in suspension culture in vitro was one of the other characteristics of pluripotent ES cells. These embryoid bodies could differentiate into various cell types in vitro thus indicating that the stem cells had pluripotency. There were two ways to discover whether these cultured cells have the differentiation ability. One was to remove LIF and to culture cells under the condition of presence of feeder layer cells and the other was to culture cells in condition absent of LIF and feeder layer cells. The fourth passage CES cells were removed and reseeded in ES cell medium devoid of LIF and feeder layer cells. 2-3 days later some floating organized structures were be detected and then these EB-like structures spontaneously differentiated into several kinds of cells such as fibroblast-like cells, epithelioid cells as shown in Figure 6. At the same time percentage of alkaline phosphatase positive colonies was much less than normal. The proliferation of these cells was

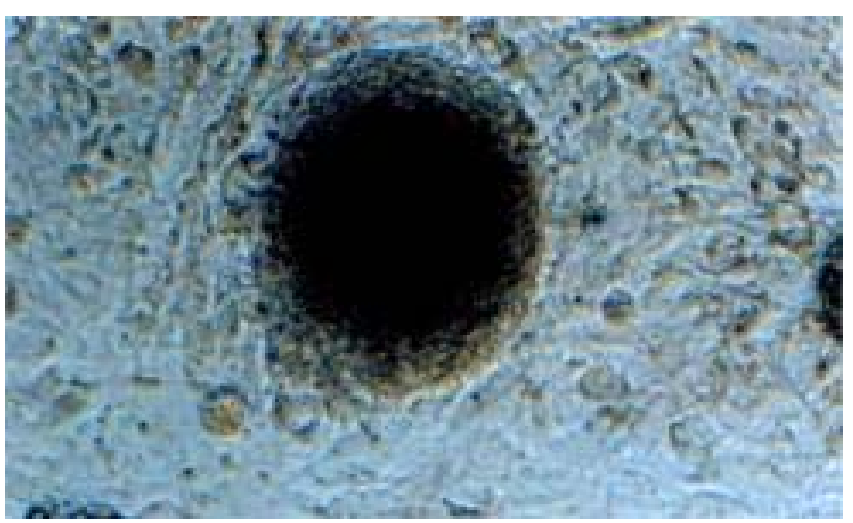

Figure 7. The fourth genetation chicken ES cells (250×).

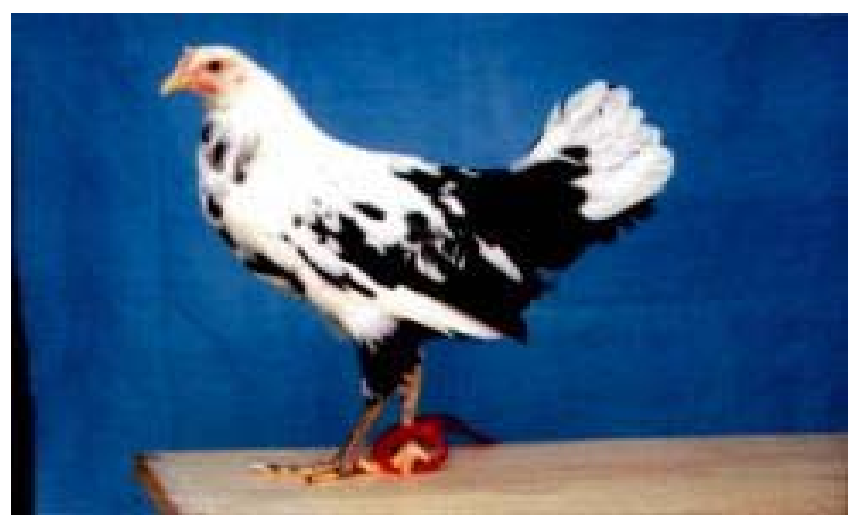

Figure 8. Chimeric chick.

slow, and a long time later differentiative cells appeared if the CES cells were cultured on feeder layer cells and with ES cell medium devoid of LIF.

\section{Production of chimeras}

The third and the fourth passage cells were injected into the subgerminal cavity of Hailan recipient embryos at stage $\mathrm{X}$ to test the in vivo differentiation potency of the cultured blastodermal cells. 269 Hailan eggs were manipulated for these series of experiments. Among these eggs, 186 eggs were injected by the third passage CES cells and 13 chicks were hatched and 3 chicks had black patches of feathers. The other 83 eggs were manipulated with fourth passage cells and 9 chicks were hatched and two chicks were chimeras (Figure 8). The amount of black patching varied among the five chicks. These date thus demonstrated that some of the cultured CES cells were able to differentiate into and contribute to various tissues in vivo. So far, further test is being done so we cannot show that these cells are able to provide germ-line transmission.

\section{DISCUSSION}

Mouse ES cells were characterized by their round and 
small shape, by their large nucleus and the strong endogenous alkaline phosphatase activity similar to the ECderived cells. The CES colonies were round, oval, well delineated and multi-layered tightly packed (Figure 7) in our chicken blastodermal cultures. In these cells there a lot of yolk particle; thus they exhibited black color under microscope. The typical ES-like properties were further supported by the presence of alkaline phosphatase activity and the capacity of differentiation in vitro. As soon as MES cells entered differentiation they rapidly lost their characteristic morphology and their alkaline phosphatase activity. The same changes were also observed in the CES cells culture when the CES cells were induced to differentiate by devoid of LIF and feeder layer cells. The production of chimeras strongly suggested that cells derived from blastoderm of chicken embryos shared ES cells features.

When comparing different natures of feeder cells, it appeared that the inactivated PMEF feeder cells provided the most regular, reproducible morphology and highest number of alkaline phosphatase positive colonies. With the continual passing and repeated freezing and thawing, the ability of SNL cells to secrete the LIF and to support the growth of CES cells was declined. Yang (1994) demonstrated that PCEF cells also could produce some LIFlike factor to sustain the growth of pluripotent cells. Mouse ES cells had been successfully co-cultured on mitotically inactivated PCEF cells for 10 passages. But the secreting mechanism was not clear and the result was not very stable.

Cell-dispersed liquid such as trypsin could stimulate the dispersion of cells. It could reversibly untie the cell microfilament and could activate the separate of cells. However, on one hand the reaction between blastodermal cells in stage $\mathrm{X}$ was so faint that we could get dispersed cells easily only by gentle aspiration, and on the other hand trypsin also had some harmful effect on cells. So it was better not to use trypsin in primary culture, while we must use trypsin to undo the stronger and stronger effect between cells for subculture.

The culture of AKP positive CES cells was dependent upon specific growth factors and cytokines like basic fibroblast growth factor (bFGF), stem cell factor (SCF) and leukemia inhibitory factor (LIF). SCF played an important role in migration, adhesion, proliferation and survival of primordial germ cells and spermatogonia during testicular development. It could increase DNA synthesis so enhance the growth of CES cells, and its ability to promote proliferation and to prevent differentiation was reinforced by the addition of LIF. LIF was able to prevent ES cells from differentiating and therefore to maintain these cells in an undifferentiated state. It could suppress programmed cell death of ES cells in culture. Certain feeder cells such as STO cells and embryonic fibroblasts cells could produce
LIF to stimulate ES cells growth. Importantly ES cells maintained in LIF retained the ability to form germ-line chimeras, which indicated that LIF was sufficient to maintain the developmental potential of ES cells. Besides LIF and SCF, bFGF was absolutely required for the longterm culture of CES cells. bFGF had been previously reported to stimulate proliferation and prevent differentiation of murine EG cells and PGCs. In this work the result showed that when these three factors were added singly, none of the factors significantly increased the number of positive colonies. However, the combination of them strongly enhanced the number of alkalinephosphatasepositive colonies.

The conclusion was that the optimum culture conditions to maintain chicken embryonic stem cells as totipotent cells included the take of cells of area pellucida from stage $\mathrm{X}$ embryos, the use of primary mice embryonic fibroblast (PMEF) cells as feeder cells and the combination of h-LIF, $\mathrm{mSCF}, \mathrm{bFGF}$ in the medium. Now these cultured cells have been maintained for four passages and exhibit features that are similar to those of MES cells such as typical morphology, alkaline phosphatase activity etc. These cells we have got also can differentiate into several kinds of cells; in the end five chimeras have been produced.

\section{ACKNOWLEDGMENTS}

We thank Dr Zhengruizhen for her kindly providing of SNL cells and Dr. Tanjinghe for his continuous assistance. We thank Dr Yanyanchun for her careful editing and helpful comments. We also had help form Liaocheng Jiaming breeding chicken farm. This work was supported by grants from Shandong Provincial Natural Science Foundation (No. Z99D03).

\section{REFERENCES}

Evans, M. J., M. H. Kaufman. 1981. Establishment in culture of pluripotential cells from mouse embryos. Nature 292:154-156.

Nichols, J., E. P. Evans and A. G. Smith. 1990. Establishment of germ-line competent embryonic stem (ES) cells using differentiation inhibiting activity. Development. 110:13411348.

Wheeler, M. B. 1994 Development and validation of swine embryonic stem cells: a review. Rep. Fertil. Dev. 6:563-568.

First, N. L., M. M. Sins, S. P. Park and M. J. Kent-First. 1994. Systems for production of calves from cultured bovine embryonic cells. Rep. Fertil Dev. 6:553-562.

Piedrahita, J. A., D. J. Murray, M. M. McGloughlin, G. B. Anderson and A. M. Oberbauer. 1999. Isolation and genetic modification of pluripotent/totipotent cell lines. 10. Transgenic animals in research.

Giles, J. R., X. Yang, W. Mark and R H. Foote. 1993. Pluritotency of cultured rabbit inner cell mass cells detected by isozyme analysis and eye pigmentation of fetuses following injection 
into blastocyst or morulae. Mol. Rep. Dev. 36:130-138.

Sun, L., C. S. Bradford, C. Ghosh, P. Collodi and D. W. Barnes. 1995. ES-like cells cultures derived from early zebrafish embryos. Mol. Mar. Biol. Biotechnol. 4,193-199.

Thomson, J. A., J. Kalishman, T. G. Golos, M. Durning, C. P. Harris, R. A. Becker and J. P. Hearn. 1995. Isolation of a primate embryonic stem cell line. Proc. Natl. Acad. Sci. USA. 92:7844-7848.

Thomson, J. A., J. Itskovita-Eldor, S. S. Shapiro, M. A. Waknitz, J. J. Swiergiel, V. S. Marshall and J. M. Jones. 1998. Embryonic stem cell lines derived from human blastocysts. Science. 282:1145-1147.

Shamblott, M. J., J. Axelman, S. Wang, E. M. Bugg, J. W. Littlefield, P. J. Donovan, P. D. Blumenthal, G. R. Huggins and J. D. Gearhart. 1998. Derivation of pluripotent stem cells from cultured human primordial germ cells. Proc. Natl. Acad. Sci. 95:13726-13731.

Petitte, J. N., M. E. Clark, G. Liu, A. M. Verrinder Gibbins and R. J. Etches. 1990. The production of somatic and germline chimeras in the chick by transfer of early blastodermal cell. Development. 108:185-190.

Natio, M., M. Watanabe, M. Kinutani, K. Nirasaxa and T. Oishi. 1991. Production of quail-chick chimaeras by blastodermal cell transfer. Br. Poult. Sci. 32:79-86.

Thoraval, P., F. Lasserre, F. Coudert and G. Dambrine. 1994. Somatic and germline chicken chimeras obtained from brown and white leghorns by transfer of early blastodermal cells. Poult. Sci. 73:1897-1905.

Ono, T., S. L. Muto and I. M. Mizutan. 1994. Production of quail chimera by transfer of early blastodermal cells and its use for transgenesis. J. Poult. Sci. 31(2):119-129.

Kino, K., B. Pain, S. P. Leibo. 1997. Production of chicken chimeras from injection of frozen-thawed blastodermal cells. Poult. Sci. 76:753-760.

Etches, R. J., M. E. Clark, L. Aajchowski, B. Speksijder, A. M. Verrinder Gibbins, K. Kino, B. Pain and J. Samarut. 1997. Manipulation of blastodermal cells. Poult. Sci. 76:1075-1083.

Gao, S. P., W. H. Shi and Y. Qin. 2000. Production of feeder layer used for isolation and culturing of embryonic stem cells, J. Lab. Anim. Sci. Sinica 10(2):78-81.
Pain, B., M. E. Clark, M. Shen, H. Nakazawa, M. Sakurai, J. Samarut and R. J. Etches. 1996. Long-term in vitro culture and characterization of avian embryonic stem cells with multiple morphogenetic potentialities. Development. 122:2339-2348.

Eyal-Giladi, H., S. Korak. 1976. From cleavage to primitive streak formation: a complementary normal table and a new look at the first stages of the development of the chick; I. General Morphology Dev. Biol. 49:321-337.

Du, L. X. and S. G. Li. 1998. Make chimeric chicken by transfer of early balstodermal cells. J. Shandong Agriculture University. 29(2):231-232.

Du, L. X. and C. G. Yin. 2002. Culture of chicken of stage X blastoderm cells. Acta. Zool. Sin. 48(4):549-553

Aajchowski, L. D., R. J. Etches. 2000. Transgenic chickens: past, present, and future. Avian and Poult. Biology Rev. 11(2):63-80.

Roger, A. P. 1994. Studies of in vitro differentiation with embryonic stem cells. Rep. Fertil. Dev. 6:543-552.

Uchida,M., T.Tokunaga, K.Niwa, and H.Imai, 1995 Effects of feeder cells and growth factors on the proliferation of mouse primordial germ cells. Theriogenology.

Yang, Z., J. N. Petitte. 1994. Use of avian cytokines in mammalian embryonic stem cell culture. Poult. Sci. 73:965-974.

Petitte, J. N., L. Karagenc. 1996. Growth factors during early events in avian embryo-development. Poult. Avian. Biol. Rev. 7:75-87.

Pesce, M., M. G. Farrace, M. Piacentini, S. Dola and M. De Felici. 1993. Stem cell factor and leukemia inhibitory factor promote primordial germ cell survival by suppressing programmed cell death (apoptosis). Development 118:1089-1094.

Williams, R. L., D. J. Hilton, S. Pease, T. A. Willson, C. L. Stewart, D. P. Gearing, E. F. Wagner, D. Metcalr, N. A. Nicola, and N. M. Gough. 1988. Myeloid leukaemia inhibitory factor mainjtains the developmental potential of embryonic stem cells. Nature 336:684-687.

Resnick, J. L., M. Ortiz, J. R. Keller and P. J. Donovan. 1998 Role of fibroblast growth factors and their receptors in mouse primordial germ cell growth. Biology of Reproduction. 59:1224-1229. 